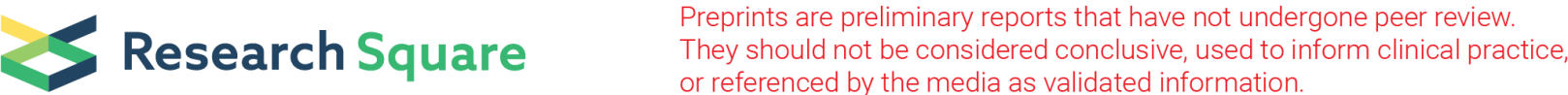

\section{Demethylation of Secreted Frizzled-Related Protein2( SFRP2) Promoter Upregulates Wnt/ $\beta$ - Catenin Activity in Endometriosis}

Mei Yang

Xiangyang Central Hospital

Chunfan Jiang

Xiangyang Central Hospital

Lin Li

Xiangyang Central Hospital

Xiaojie Huang

Xiangyang Central Hospital

Hui Xing

Xiangyang Central Hospital

Li Hong ( $\nabla$ drwhdxrmyyhongli@163.com )

Renmin Hospital of Wuhan University: Wuhan University Renmin Hospital https://orcid.org/0000-00027806-3541

\section{Research Article}

Keywords: SFRP2, Endometriosis, Wnt pathway, Demethylation

Posted Date: November 11th, 2021

DOI: https://doi.org/10.21203/rs.3.rs-1048240/v1

License: (c) (i) This work is licensed under a Creative Commons Attribution 4.0 International License.

Read Full License 


\section{Abstract}

Wnt/ $\beta$-catenin signalling contributes to the metastasis and invasion in the etiology and pathogenesis of endometriosis (EMS), but why the WNT pathway is dysregulated in EMS remains unclear. This study aimed to explore the effects of demethylation of SFRP2 promoter on the Wnt/ $\beta$-catenin activity in EMS. Aberrantly methylated-differentially expressed genes were identified from GEO database microarray data. 5 ectopic endometrium and 5 normal endometrium were get, subsequently, ectopic endometrium epithelial cells (EEECs) and normal endometrium epithelial cells (NEECs) were isolated in vitro. MSP, BSP, luciferase reporter assay, Lentivirus infection of high expression of SFRP2 gene vector, low expression of DNMT1 gene vector, and 5-Aza stimulation, RT-PCR and western blot were performed in the tissues or cells. It was found that compared with the normal endometrium and NEECs, the RNA and protein expression levels of SFRP2 were significantly increased while the SFRP2 promoter was demethylated in ectopic endometrium and EEECs. The 5-Aza treatment significantly upregulated SFRP2 mRNA and protein levels in EEECs. Furthermore, after the knockdown of DNMT1 expression, the demethylation of the SFRP2 promoter and upregulation of SFRP2 mRNA and protein in EEECs were observed. Meanwhile, the expression of lentivirus carrying SFRP2 CDNA up-regulates the activity of Wnt signaling and the protein expression of $\beta$-catenin in EEECs. In summary, the increased SFRP2 expression-induced Wnt/ $\beta$-catenin signaling due to the demethylation of the SFRP2 promoter plays an important role in the pathogenesis of EMS, suggesting that SFRP2 might be a therapeutic target for EMS treatment.

\section{Introduction}

As a common disease, EMS affects about $5 \%-10 \%$ of women of reproductive age, which causes decreases in life quality and is accompanied by symptoms such as pelvic pain and affects more than $10 \%$ of reproductive-age women[1, 2]. Several classical theories including Mül-Lerianosis, retrograde menstruation, and coelomic metaplasia have been proposed to elucidate the pathogenesis of EMS, but the molecular mechanism is still unknown[3, 4].

Many differences were found in gene expression profiles between EMS samples and the normal endometrium tissue samples[4-6]. Nowadays, microarray technology has become a mature and stable technology, and during the last decade's bioinformatics analysis has been widely used to identify general genetic of etiology and pathogenesis in many malignant tumors [7-9], but there have been few reports of EMS. Several gene expression microarrays could be used for identifying differentially expressed genes(DEGs) and pathways in EMS [10-13], and it will provide a possible breakthrough for the diagnosis, gene-targeted drug development, and evaluation of the therapeutic effect of EMS. Using bioinformatics analysis of GEO database microarray of differentially expressed genes of EMS vs normal endometrial tissue, 48 genes were found upregulated, of which, SFRP2 (secreted frizzled-related protein 2) was significantly upregulated, and was seldom reported in EMS, so it was selected by us for further study.

Characterized histologically by dense fibrous tissue consisting, EMS is researched by many scholars and it was found that treatment with Wnt3a significantly increased the proliferation and migration of 
endometrial cells in patients with EMS, and significantly enhanced the expression of fiber marker genes, such as a-smooth muscle actin, type I collagen, connective tissue growth factor and fibulin, which were closely related to the contraction of collagen gel[14, 15]. Some studies focus on the effect of endometrial cells-mediated collagen gel contraction on EMS[16]. After treatment with Wnt3a, the contraction of collagen gel $I$ in the endometrial cells in normal endometrium was increased to a level comparable to that in EMS patients[17-19]. Wingless MMTV integration site family (Wnt) signaling is an early event in some tissue carcinogenesis, there is evidence that the Wnt signaling pathway also plays a role in the etiology of EMS[20-22].

SFRP2 is a member of the various secreted frizzled-related protein (SFRP) family proteins, which are one of the main regulator proteins members of the Wnt pathway, and in different tissues, it could have the opposite activity. Studies have shown that SFRP2 can act as an agonist or antagonist for Wnt signaling[23, 24]. T. Heinosalo found that after SFRP2 knockout, cell proliferation, and $\beta$-catenin protein expression in primary cultured cells with EMS significantly reduced, suggesting that in EMS, SFRP2 acts as an agonist for the Wnt signaling pathway and. stimulates lesion growth[24, 25].

Being considered as a heritable change in gene expression, epigenetics covers abnormal DNA methylation[26], abnormal non-coding RNA, altered histone modification after translation, etc\among which abnormal DNA methylation is most widely studied[27, 28]. Epigenetic modifications were reported to play a role in the pathogenesis of EMS in recent years[29-33]. Some scholars found that the epigenetic mechanisms including DNA methylation and histone modification closely related to the expression of estrogen receptors and progesterone receptors in patients with EMS[29]. Other scholars evaluated and compared the methylation pattern of Human Homeobox clusters in normal, eutopic (endometrium in the uterine cavity of the EMS patients), and ectopic endometrial tissues, a conserved pattern of methylation alterations in EMS tissues was observed for most of the investigated genes (56 of 84) which indicating epigenetic changes in EMS[32]. In different diseases, SFRP proteins were reported correlating with the Wnt pathway, and their expression was regulated by methylation[24, 34]. For example, SFRP2 is reported to be closely related to Wnt and regulated by methylation in nasopharyngeal carcinoma[35]. This study aimed to explore the effects of demethylation of SFRP2 promoter on the Wnt/ $\beta$-catenin activity in EMS.

\section{Materials And Methods}

\section{Microarray data}

Next-generation sequencing dataset (GSE135485) and methylation profiling dataset (GSE47359) were obtained from the GEO database. GSE135485 included 54 EMS samples and 4 normal endometrium tissue samples, based on GPL21290 Illumina Human HiSeq 3000 platform. GSE47359 consisted of 3 EMS samples and 6 normal endometrium tissue samples, based on the GPL8490 Illumina Human Methylation 27 platform. 
On data processing and identification of DEGs, R software (ver. 3.6.3, https://www.rproject.org/) were used to identify DEGs and differentially methylated genes(DMGs). The matrix file for GSE135485 was downloaded from https://www.ncbi.nlm.nih.gov/geo/query/acc.cgi?acc=GSE135485 and then gene IDs conversion was conducted with strawberry-Perl-5.30.0.1. The data normalization was done with the limma package and then processed with the edgeR package to get DEGs. The cutoff value of DEGs was

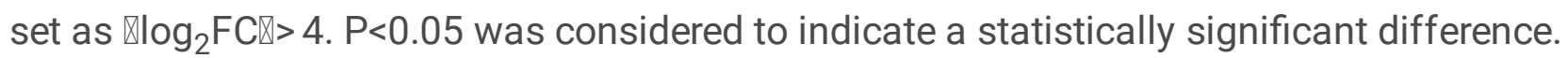

\section{Differential methylation genes (DMGs) identification}

The HumanMethylation 27 BeadChip array, covers approximately 27578 CpG sites at different gene regions, embodying the upstream region of the transcriptional start site, 5'untranslated region, exons, 3'untranslated region. The matrix file for GSE47359 was downloaded from ftp://ftp.ncbi.nlm.nih.gov/geo/series/GSE47nnn/GSE47359/matrix/.

The Champ package of $\mathrm{R}$ was used for the identification of $\mathrm{CpG}$ sites and DMGs with the threshold $\mathrm{P}<$ 0.05 and $\mathbb{\operatorname { l o g } _ { 2 }} \mathrm{FCQ}>0.2$. The Champ package is a highly integrated methylation analysis tool, matching the corresponding DMGs with the most differentially methylated $\mathrm{CpG}$ sites. A Venn diagram was used to illustrate the intersection between DEGs and DMGs. As a result, upregulated hypomethylated genes were listed.

\section{GO term and KEGG pathway enrichment}

Online analysis tool DAVID was used to conduct Gene ontology (GO) Enrichment Analysis of DEGs into the Cell Components(CC), Molecular Functions(MF), and Biological Processes(BP). All $p$ values $<0.05$ were considered to be statistically significant.

\section{Ethical approval and Patient recruitment}

The protocol of the present study was approved by the Medicine Ethics Committee of Xiangyang Central Hospital (Approved Code: 2017-004), and was carried out in accordance with the Declaration of Helsinki. An informed consent was signed by each recruited patient. This study was initiated on November 11th, 2019 and terminated on April 20th, 2021.

All of the women recruited in this study were being at child-bearing age and underwent laparoscopic surgery at the Department of Gynecology of Xiangyang Central Hospital. Five women with endometriosis were recruited before surgery. All these women had not received $\mathrm{GnRH}-\mathrm{a}$ agonist or hormones treatment for at least six months and were preoperative diagnosed as an ovarian cyst. They were aged between 24 and 39 years old, mean \pm SD $(32.12 \pm 4.90)$ years; Each case of endometriosis was staged during the operation according to the revised American Fertility Society classification of endometriosis (rAFS) and subsequently confirmed by histology. Among them, two were in rAFS staging III and the other three were 
in rAFS staging IV. All these patients were in the secretory phase of the menstrual cycle. Ectopic endometrium from the ovarian cyst of these 5 patients were obtained by laparoscopy.

Five women undergoing tubal ligation for sterilization were recruited as controls. All these five patients were aged between 28 and 40 years old, mean \pm SD (34.60 \pm 4.38$)$ years. No minimal endometriosis was found in these control subjects and no hormones treatment for at least six months. All these women were in the secretory phase of the menstrual cycle. Normal endometrium were obtained by curettage during tubal ligation operation.

\section{Cell culture}

According to our previous study[36], tissues were washed with sterile Hank's Balanced Salt Solution(HBSS, phenol-red-free) three times, then minced into pieces of approximately $1 \mathrm{~mm}^{3}$ and digested in $10 \mathrm{ml}$ of HBSS containing $10 \mathrm{U} / \mathrm{ml}$ DNase I (Sigma) and type IV collagenase (0.03\%; Sigma, St. Louis, MO) for 40 min at $37^{\circ} \mathrm{C}$. The supernatant was kept and epithelial cells and stromal cells in it were separated by differential centrifugation [21]. To repurify the endometrial cells, the selective attachment was carried out [22]. The endometrial cells were cultured in phenol-red-free DMEM/Ham's F12 (Invitrogen, Carlsbad, CA) supplemented with $10 \%$ v/v fetal bovine serum (FBS; Invitrogen). Next, they were subjected to differential trypsinization and attachment for further purification. Finally, the primary epithelial cells were plated $(2 \times 104 \mathrm{cell} / \mathrm{ml})$ in dishes in a culture medium as mentioned above. The detect the phenotypic characterization and ensure the purity of endometrial cell $>95 \%$, the primary epithelial cells were tested by dyeing of vimentin and PCK.

\section{Western blot}

Western blot was performed according to our previous study [36] using primary anti-bodies against human SFRP2 (rabbit polyclonal, \#HPA002652, Sigma-Aldrich, Merck, USA), anti- $\beta$-catenin (\#ab6302, Abcam), DNMT1 (\#ab13537, Abcam), and mouse monoclonal anti- $\beta$-actin (\#A5441, Sigma-Aldrich) antibodies. The intensities of the protein bands were measured using the Image $(1.49 \mathrm{v})$ program.

\section{5-Aza-2'-deoxycytidine (Aza) treatment of EEECs}

As deoxycytidine analogs, 5-Aza-CdR can be irreversibly mixed into DNA for synthesis, thus reducing the ability of DNA to accept methyl under the action of methyltransferase (DNMT). Meanwhile, 5-Aza-CdR forms a covalent complex with DNA methyltransferase (DNMT), reducing the activity of DNMT. And we want to decrease the methylation rate of the promoter of SFRP2 by using this drug. The EEECs were grown and treated with 1 uM of 5-Aza (Sigma-Aldrich \#CAS 2353-33-5) for 3 days for the inhibition of DNA methyltransferase activity.

\section{Real-time RT PCR}

Total RNA was isolated from EMS tissues and EEECs utilizing the TRIzol reagent (Invitrogen, Shanghai, China), and all cRNA transcripts were generated using a primeScript ${ }^{\text {TM }}$ RT kit (Qiagen, Hilden, Journal of 
Molecular Histology 1 Germany). All primers (Sangon Biotechnology, China) were listed as fellows: SFRP2, 5'-TGGGGGAAACGGTCGCACTC-3', and 5'-GGCCACGAGACCATGAAGGAGG-3'. $\beta$-catenin, 5'AAAGCGGCTGTTAGTCACTGG-3' and 5'-CGAGTCATTGCATACTGTCCAT-3'. The qPCR was performed in triplicate to determine the relative levels of the target mRNA using SYBR premix Ex Taq ${ }^{\text {TM }}$ Green II (Takara) in the CFX96 Touch sequence detection system (Bio-Rad, Hercules, CA, USA). Quantitative real-time PCR was conducted ABI 7500 Real-Time PCR System(Applied Biosystems/Life Tech).

\section{Luciferase reporter assay}

To detect the Wnt/ $\beta$-catenin activation in EEECs, TOP/FLASH and FOP/FLASH reporter gene system (GenePharma Company, Shanghai) were selected to test the Wnt signaling pathway and the Promega dual-luciferase reporter gene assay system was used to measure the reporter activity. TOP/FOP values were used to represent the result. A higher value of TOP /FOP indicates a stronger Wnt pathway activity.

\section{Methylation-specific PCR (MSP)}

Genomic DNA from 5 ectopic endometrium and 5 normal endometrium was isolated using the DNA Extraction Kit (Sangon Biotech, Shanghai, China). In the 50ul system, DNA (2-5 $\mu \mathrm{g})$ was denatured by $\mathrm{NaOH}$ (final concentration $0.2 \mathrm{~mol} / \mathrm{L}$ ) at $37^{\circ} \mathrm{C}$ for $10 \mathrm{Min}$. Add $30 \mu \mathrm{L}$ of $10 \mathrm{mmol} / \mathrm{L}$ hydroquinone and $40.5 \%$ sodium bisulfite to mix well, then incubate for $16 \mathrm{~h}$ in the condition of air isolation and out of light. The modified DNA passed by a DNA purification column and then eluted by water. At room temperature, it was modified with $\mathrm{NaOH}$ (the final concentration was $0.3 \mathrm{~mol} / \mathrm{L}$ ) for $5 \mathrm{~min}$, and then precipitated with ethanol. Dissolve the DNA in $20 \mu \mathrm{L}$ water, stored at $-20^{\circ} \mathrm{C}$. Two pairs of specific primers were used to amplify the same nucleotide sequence of the tested gene using methylated primer pairs (M) $5^{\prime}-$ GGAGTTTTTCGGAGTTGCGC-3' and 5'-CTCTTCGCTAAATACGACTCG-3', or unmethylated primer pairs (U) 5'-GTTGGAGTTTTTTGGAGTTGTGT-3' and 5'-CTCTCTTCACTAAATACAACTCA-3'. The amplified products were detected by DNA agarose gel electrophoresis and analyzed by gel scanning.

\section{Bisulfite sequencing PCR}

Genomic DNA from 5 ectopic endometrium and 5 normal endometrium was isolated using the DNA Extraction Kit (Sangon Biotech, Shanghai, China). According to the manufacture's instruction, and bisulfite modification was performed with the EZ DNA Methylation Gold Kit (Tianmo Technology, Beijing, China). Primer(Sangon Biotechnology, China) sequences for bisulfite sequencing were listed as follows: forward(M818-F)5'-TTTATGTTTGGTAATTTAGTAGAAATTT-3' and reverse (M818-R) 5'-

ATTTTACRTTAAAAATACCCCTCAC-3'. This area was 302-bp fragments including $28 \mathrm{CpG}$ dinucleotides.

The PCR conditions were: pre-denaturation at $95^{\circ} \mathrm{C}$ for $3-5 \mathrm{~min}$, denaturation at $94^{\circ} \mathrm{C}$ for $30 \mathrm{~s}, 55-60{ }^{\circ} \mathrm{C}$ for $30 \mathrm{~s}$, and $72^{\circ} \mathrm{C}$ for $30 \mathrm{~s}, 35$ cycles totally. Then, the sequence containing the SFRP2 sequence was sequenced(Sangon Biotech, Shanghai, China).

\section{Plasmid construction and lentivirus production}


The human SFRP2 lentiviral vectors were purchased from GenePharma and transfected EEECs according to standard manufacturer protocols. Furthermore, lentiviral vectors to knockdown DNMT1 expression were generated by the GenePharma Company, (Shanghai), and the interfering sequence is as follows: DNMT1-Homo-2664 GGAGCTGTTCTTGGTGGATGA. Three kinds of infection sequence were tested in the preliminary experiments, and one is useful as mentioned above. Post-infected cells were cultured for one week consecutively and lentivirus infection condition of target cells were determined by observing the expression time and intensity of GFP. To screen the stably transfection clusters, at the basis of transient infection, puromycin with minimum lethal concentration lasts for at least 4 days.

\section{Immunohistochemistry}

A cohort of 84 formalin-fixation paraffin-embedded specimens (FFPE), including 28 EMS ectopic endometrium, 28 eutopic endometrium and 28 normal endometrium were retrieved from Xiangyang Central Hospital from 2006 to 2020 with necessary clinical information. 28 eutopic endometrium and ectopic endometrium were get from 28 ovarian endometrial cyst patients which were aged between 25 and 43 years old, mean \pm SD $(35.05 \pm 8.70)$ years; normal endometrium patients were aged between 29 and 48 years old, mean \pm SD $(41.80 \pm 6.22)$ years. All the cases were reviewed by two senior pathologists separately again to ensure the diagnosis accuracy.

Immunohistochemical staining for SFRP2 was performed with 3- $\mu$ m-thick sections using the Ventana Benchmark ULTRA automated staining system (Ventana Medical Systems, Tucson, AZ) according to the manufacturer's protocol. SFRP2 (Abcam), the primary antibodies were added on the cell sections for two hours, Sections were incubated with a secondary antibody and visualized with 3, 3'-diaminobenzidine tetrahydro-chloride (DAB; Golden Bridge, Beijing, China). Sections were then subjected to nuclear counterstaining (blue staining) with hematoxylin. Two investigators were asked to review and score the anti-SFRP2 staining on the stained sections by adding the percentage score with the intensity score. Staining intensity was scored as 0 (negative), 1 (weak), 2 (moderate) and 3 (strong), while staining percentage was scored as 0 ( $<10 \%$ staining), 1 (11-25\% staining), 2 (25-75\% staining) and 3 ( $\geq 75 \%$ staining). And these two fractions were added together, score 0-3: low; 4-6: moderate; 7-9: high.

\section{Statistical analysis}

All the experiments were repeated at least three times. SPSS 13 software was used for statistical analysis of all experimental data. The data were normally distributed. The comparison between the two groups was estimated by Student's t-test. A p-value $<0.05$ was considered significant. Chi-square was used in the Statistical analysis of immunohistochemistry data.

\section{Results}

\section{Identification of aberrantly methylated-differentially expressed genes}


A total of $3215 \mathrm{CpG}$ sites were found and associated with the profile of differentially expressed genes from a microarray analysis from the GEO database (Fig. 1). After the GO analysis of the low methylation expression, the functions of these hypomethylated genes were explored in several important cell processes, including repressor, secreted, and signaling (Tab. 1).

\section{Screening for DEGs}

To identify DEGs in EMS compared to healthy controls, one next-generation sequencing dataset (GSE135485) with the 54 EMS and 4 healthy controls has been analyzed using the linear modeling approach. A total of 134 DEGs were identified after the screening, of which 48 genes were upregulated and 86 were downregulated $\left(\mathbb{l _ { 0 }} \log _{2} \mathrm{FC} \backslash>4, \mathrm{p}<0.05\right)$ (Fig. 2). The downregulation genes were not the research focus, so we did not present them here. Among upregulation genes, 48 genes $\left(\mathbb{\operatorname { l o g } _ { 2 }} \mathrm{FC} \rrbracket>5\right.$, $p<0.05)$ were selected for subsequent bioinformatic analysis. The significant terms of $G 0$ enrichment analysis performed by DAVID were illustrated in table2. There are two upregulation and demethylation genes(Fig 3). Among them, SFRP2 was seldom described in EMS yet. Therefore, we further verified the expression of SFRP2 and study its function in the development of EMS.

\section{Increased SFRP2 expression in EMS tissues and EEECs (ectopic EMS endometrium cells)}

The immunohistochemistry experiments' results of normal endometrium(Fig. 4A), eutopic endometrium(Fig. 4B) and ectopic endometrium(Fig. 4C) were presented in Figure 4. According to the Chisquare analysis of the results, the SFRP2 expression in the eutopic endometrium samples showing no differences compared with normal endometrium samples, $c 2=2.938 \llbracket p=0.087>0.05$. So only normal endometrium samples were used in the following analyses. From Chi-square analysis, there was significant differences between the SFRP2 expression in these three groups, $c 2=17.907, p=0.000<0.001$. So compared with the normal endometrium and eutopic endometrium, the protein expression levels of SFRP2 were significantly increased in EMS. And through the immunohistochemistry experiments, it was found that SFRP2 was located in the cytoplasm of EEECs and was yellowy-brown(Fig. 4D).

The SFRP2 protein expression in EMS vs normal endometrium and EEECs vs NEECs assessed using western blot were exhibited in Fig. 5A and Fig. 5B. All the experiments were repeated three times, the results of statistical analysis were shown in Fig. 5C and Fig. 5D. It was found that SFRP2 protein levels were significantly upregulated in EMS, compared with normal endometrium ( $P=0.003<0.01$; Fig. $5 C$ ), meanwhile, compared with NEECs, the protein expression levels of SFRP2 in EEECs were significantly increased $(P=0.0118<0.05$; Fig. 5D).

Next, through the RT-PCR, it was found that compared with NEECs, the mRNA expression levels of SFRP2 in EEECs were significantly increased $(P<0.001$, Fig. $5 E)$, moreover, that SFRP2 mRNA expression levels were significantly upregulated in EMS, compared with normal endometrium $(P=0.0044<0.01$, Fig. $5 F)$.

\section{Demethylation of the SFRP2 promoter in EMS}


From the MSP data, it was found that compared with that in normal endometrium, the SFRP2 promoter region was hypomethylated in EMS, $(P<0.0001$, Fig. $6 A)$. To further investigate whether the activation of SFRP2 is related to the methylation status of the promoter, Bisulfite sequencing PCR was used in EEECs and NEECs. Direct sequencing analysis of a 302-bp fragment including $28 \mathrm{CpG}$ dinucleotides in the SFRP2 promoter was performed. Differential methylation was observed in $28 \mathrm{CpG}$ dinucleotides of the promoter in these two kinds of cells. We found that the percentage of methylated $\mathrm{CpG}$ dinucleotides in EEECs and NEECs was 41.8 and $77.6 \%$, respectively $\mathbb{P}=0.002$ (Fig. 6B), This suggests that hypomethylation of the SFRP2 promoter in EMS.

\section{SFRP2 was upregulated due to the reduced methylation status of the promoter}

After the treatment with 5-Aza-2'-deoxycytidine, from MSP data, it was found that the level of SFRP2 promoter methylation in EEECs was significantly reduced $(P<0.01)$ (Fig. $6 C)$, and the mRNA $(P<0.0001)$ levels of SFRP2 were significantly increased at the same time (Fig. 6D) in EEECs.

DNA methyltransferase(DNMT) is a pivotal isozyme for DNA methylation. To further understand the influence of promoter methylation on SFRP2 expression, the depletion of DNA methyltransferase(DNMT) was performed in EEECs. There were several DNMT, and through preliminary experiments, it was found that the level of SFRP2 promoter methylation was significantly reduced in EEECs by DNMT1 knockout. So next we choose lentiviral vectors to knockdown DNMT1 expression in the following experiments. And it was found that the mRNA $(P<0.0001)$ levels of SFRP2 were significantly increased after knockdown of DNMT1 in EEECs(Fig. 6E),

To detect the SFRP2 protein expression in EEECs, western-blot was used. After the treatment with 5-Aza2'-deoxycytidine, it was found that the levels of SFRP2 protein were significantly increased(Fig. 6F). Meanwhile, the levels of SFRP2 protein were significantly increased after knockdown of DNMT1.(Fig. 6G). From these two experiments, it was observed that DNA hypomethylation in the promoter region promoted the upregulation of SFRP2 in EEECs.

\section{The regulation of the Wnt signaling pathway after ectopic expression of SFRP2 in EMS}

After the transfection of SFRP2 cDNA into EEECs, it was found that SFRP2 mRNA levels were upregulated significantly $(P<0.0001$, Fig. $7 A)$. Meanwhile, Wnt/ $\beta$-catenin signaling activity was also increased $(P<0.0001$, Fig. 7B) from the Luciferase reporter assay. The mRNA $(p<0.0001$, Fig. $7 C)$ and protein (Fig. 7D) expression of $\beta$-catenin, the downstream target gene, were increased after SFRP2 cDNA transfection.

\section{Discussion}

Because of the complexity of biological traits and high heterogeneity, inadequate knowledge about mechanisms at the molecular and cellular levels [11,37], the pathogenesis of EMS has yet to be fully elucidated. With the great development of microarray technology, the general genetic alteration of EMS 
has been researched with it[38, 39]. The key gene targets to regulate the relevant signaling pathways may be found for better treatment of EMS[40, 41]. The Wnt pathway has been reported to play an important role in the pathogenesis of EMS, but the key target of Wnt pathway regulation in the development of EMS remains unclear. To find the key target for regulating the Wnt pathway leading to the occurrence and progress of EMS through bioinformatics is the aim of this study. In the present study, microarray datasets of normal endometrium tissues and EMS samples were obtained to identify the DEGs. To interpret the biological functions of these common DEGs, GO and pathway analysis based on the DAVID tool was performed. Meanwhile, to identify the alteration in gene expression and regulation, the microarray data on the DNA methylation profile for EMS was screened.

SFRP2 and GATA4, two hypomethylation-high expression genes were screened out from overlapping 48 up-regulated and 85 hypomethylation genes. Our interest focused on SPRP2. The study on the relationship between SFRP2 and EMS was rarely reported in our literature review yet. In this study, we investigated the epigenetic changes of the SFRP2 gene and their effects on SFRP2 expression, and the over-expression of SFRP2 in EEECs and the hypomethylation of SFRP2 promoter were proved. Furthermore, by targeting SFRP2 using lentivirus carrying SFRP2 CDNA, we have demonstrated the upregulation of the expression of SFRP2. And after the up-regulation of SFRP2 caused by the lentivirus, the up-regulation of the protein expression of $\beta$-catenin and activity of Wnt signaling in EEECs were observed, further confirming that SFRP2 may be an important factor in the up-regulation of Wnt signaling in EMS tissues. And the up-regulation of SFRP2 could be strengthened after the knockdown of DNMT1 or 5-Aza treatment. Therefore, we can conclude that demethylation of SFRP2 leads to activation of the Wnt/ $\beta$ catenin signaling pathway in EMS.

Some researches have found that the aberrant activation of Wnt/ $\beta$-catenin signaling significantly correlated with the pathophysiology of EMS. Some studies found that being a subunit of the cell surface cadherin protein complex, $\beta$-catenin act as an intercellular signal transducer in the Wnt signaling pathway and involve in the progress of EMS[20]. Other scholars found that under the regulation of E2, the promotion of MMP9 by Wnt signaling pathway may contribute to the metastasis, detachment, invasion, and implantation of EMS[21]. And there are still researches found that Defective endometrial stromal fifibroblasts (EMSFs) contribute to EMS, but before implantation, the activation of $\beta$-catenin was essential for the key differentiation step of EMSFs[22]. Only one research found that the mRNA levels and protein levels of b-catenin, GSK-3b, and WNT7a in EMS group, were not signifificantly different with those in control group[34]. The incompatible results may be explained by tissues selected from different menstrual cycles.

Numerous researches have devoted to reveal the regulation factors of WNT pathways. Scholars found that secreted frizzled-related proteins (SFRPs) and some other secreted proteins can competitively displace certain WNT ligands in some cancer models, and increase in SFRP levels attenuates cancer growth, particularly in breast cancer cells[20,42]. But in the researches of prostate cancer cells in vitro, the overexpression of SFRP1 promotes the growth of BPH1, whereas overexpression of SFRP4 or SFRP3 decreases the proliferation of human PC3 cells[42]. 
Only one study concern how SFRP regulated Wnt pathway in EMS, the scholars found that the increased SFRP2 expression indicates the active endometriotic stroma and epithelium, and indicates the EMS lesion borders,too. Meanwhile, they also found $\beta$-catenin and SFRP2 showed similar expression patterns, suggesting that overexpression of SFRP2 promotes the activity of Wnt signal and the growth of EMS lesions[24]. In this study, it was found that compared with the normal endometrium, the protein expression levels of SFRP2 were significantly increased in EMS. Our conclusions are similar to those of the above.[24]

Methylation of SFRPs, DACT2, DKK2, and many other regulators was frequently detected in cancers, such as nasopharyngeal carcinoma[35], Being as heritable changes in gene expression, epigenetics especially methylation of key regulators plays a critical role in carcinogenesis without alteration in DNA sequence For example, effects of certain genes with aberrant DNA methylation on HCC and mammary stem cells have been extensively reported[26], Aberrant DNA methylation could influence some tumor suppressor genes which were key genes involved in the carcinogenesis of HCC and mammary stem cells. So we wanted to detect the methylation status of SFRP2 in EMS. Interestingly, bioinformatics analysis of DMG microarray and related experiments showed that SFRP2 was significantly demethylated in EMS.

There are great clinical relevance in our study. Abnormal activation of the Wnt/ $\beta$-catenin signaling pathway may be involved in the aggressive phenotype of EMS cells[23]. Pain is a major clinical problem in patients with EMS, Wnt3a and $\beta$-catenin are upregulated in various mouse pain models, activating Wnt signaling and possibly contributing to central spinal cord conduction[37]. However, only one literature has reported the regulatory effect of SFRP on the Wnt pathway in EMS, and no literature has been found about the role of methylation on the SFRP expression in EMS. Therefore, the study of SFRP2 in EMS can provide more profound information for the development of EMS and provide new strategies for the clinical control of EMS in the future.

To determine how SFRP2 regulated the Wnt/ $\beta$-catenin signaling pathway, the expression of downstream target were detected after using some demethylation treatment. In one study, scholars found that in colorectal cancer, high levels of LEF1 were associated with reduced patient survival.[42]

The classical Wnt signaling pathway requires $\beta$-catenin to enter the nucleus and then bind to the transcription factor TCF/LEF to form a complex, which initiates the transcription of downstream regulatory genes. Scholars found that in the proliferative progenitor cells of colon crypts, the activation of a specific subset of the TCF/LEF family regulate the expression of many target genes that are normally associated with tumorigenesis[42]. Meanwhile, in other researches, it was found that after SFRP2 knockdown, cell proliferation signifificantly reduced, and there is a strong positive correlation between cell proliferation and SFRP2 mRNA expression[37, 42].

All these studies indicates that WNT signalling in EMS cannot be targeted using the same strategy of cancer, increasingly detailed understanding of WNT signalling in EMS will help us to make clinical decision. In our research, after the up-regulation of SFRP2 caused by the lentivirus, the up-regulation of 
the protein expression of $\beta$-catenin and activity of Wnt signaling in EEECs were observed, further confirming that SFRP2 may be an important factor in the up-regulation of Wnt signaling in EMS tissues.

There are obvious strengths in the present study. To date, bioinformatics analysis was rarely used in EMS and there have been few reports regarding the role of SFRP2 in the development of EMS. In this study, next-generation sequencing dataset and methylation profiling dataset were used together and differentially expressed and abnormally methylated genes were found in EMS. Further more, in this study, primary endometrial cells were isolated and cultured, and cell models of transfection were build. This is the first study which clarified the mechanism of SFRP2 demethylation and its interaction with Wnt pathway in the pathogenesis of EMS.

However, there are limitations in the present study: One is that the microarray data were not generated by the authors but from the GEO database. The second limitation of the study is the sample size was relatively small.

In conclusion, this study confirmed that SFRP2 is a novel regulatory gene in EMS. SFRP2 is activated in EMS due to promoter demethylation.Our study could provide new clues to the underlying biological mechanisms of EMS, search for new biomarkers, and further develop diagnosis and treatment methods for EMS. But this is only the first step. The specific mechanism of SFRP2 demethylation in EMS requires further investigation.

\section{Declarations}

Fund: National Natural Science Foundation of China (no. 81972449)『Hubei Provincial Natural Science Foundation of China(2019CFA016), Science and technology project of Xiangyang Central Hospital(2021C12)

Conflicts of interest: The authors declare that no conflict of interest exists.

Availability of data and material: Not applicable

Code availability: Not applicable

Authors' contributions

Designed project: HL, XH. Collected samples: YM, JCF, LL, HXJ. Analyzed data: YM, JCF. Generated figures and tables: YM, JCF. Wrote manuscript: YM. All authors read and approved the final manuscript.

Ethics approval

This study received the verification of Medicine Ethics Committee of Xiangyang Central Hospital (Approved Code: 2017-004). 
Consent to participate: Not applicable

Consent for publication: Not applicable

\section{References}

[1] Sanchez AM, Somigliana E, Vercellini P, Pagliardini L, Candiani M, Vigano P. Endometriosis as a detrimental condition for granulosa cell steroidogenesis and development: From molecular alterations to clinical impact. J Steroid Biochem Mol Biol. 2016. 155(Pt A): 35-46.

[2] Gerkowicz SA, Curtis SW, Knight AK, et al. Endometriosis, endocrine disrupters, and epigenetics: an investigation into the complex interplay in women with polybrominated biphenyl exposure and endometriosis. J Assist Reprod Genet. 2020. 37(2): 427-436.

[3] Karamian A, Nazarian H, Ziai SA, et al. Pyrvinium pamoate inhibits proliferation and invasion of human endometriotic stromal cells. Hum Exp Toxicol. 2020. 39(5): 662-672.

[4] Li R, Wu SP, Zhou L, et al. Increased FOXL2 expression alters uterine structures and functionst. Biol Reprod. 2020. 103(5): 951-965.

[5] Cui D, Liu Y, Ma J, Lin K, Xu K, Lin J. Identification of key genes and pathways in endometriosis by integrated expression profiles analysis. PeerJ. 2020. 8: e10171.

[6] Jiang J, Yu K, Jiang Z, Xue M. IL-37 affects the occurrence and development of endometriosis by regulating the biological behavior of endometrial stromal cells through multiple signaling pathways. Biol Chem. 2018. 399(11): 1325-1337.

[7] Stevens-Kroef M, Simons A, Rack K, Hastings RJ. Cytogenetic Nomenclature and Reporting. Methods Mol Biol. 2017. 1541: 303-309.

[8] Behzadi P, Ranjbar R. DNA microarray technology and bioinformatic web services. Acta Microbiol Immunol Hung. 2019. 66(1): 19-30.

[9] Leti F, Llaci L, Malenica I, DiStefano JK. Methods for CpG Methylation Array Profiling Via Bisulfite Conversion. Methods Mol Biol. 2018. 1706: 233-254.

[10] Anastasiu CV, Moga MA, Elena Neculau A, et al. Biomarkers for the Noninvasive Diagnosis of Endometriosis: State of the Art and Future Perspectives. Int J Mol Sci. 2020. 21(5).

[11] Goulielmos GN, Matalliotakis M, Matalliotaki C, Eliopoulos E, Matalliotakis I, Zervou MI. Endometriosis research in the -omics era. Gene. 2020. 741: 144545.

[12] Adewuyi EO, Sapkota Y, lec IEC, et al. Shared Molecular Genetic Mechanisms Underlie Endometriosis and Migraine Comorbidity. Genes (Basel). 2020. 11(3). 
[13] Adewuyi EO, Mehta D, Sapkota Y, et al. Genetic analysis of endometriosis and depression identifies shared loci and implicates causal links with gastric mucosa abnormality. Hum Genet. 2021. 140(3): 529552.

[14] Xu H, Yang JJ, Wang CH, Guo EY, Yang NH, Zhao Q. Effect of Wnt/ß-catenin signal pathway on of matrix metalloproteinase-7 and vascular endothelial growth factor gene expressions in endometriosis. Clin Exp Obstet Gynecol. 2016. 43(4): 573-577.

[15] Pazhohan A, Amidi F, Akbari-Asbagh F, et al. Expression and shedding of CD44 in the endometrium of women with endometriosis and modulating effects of vitamin D: A randomized exploratory trial. J Steroid Biochem Mol Biol. 2018. 178: 150-158.

[16] Pazhohan A, Danaei-Mehrabad S, Mohamad-Rezaeii Z, et al. The modulating effects of vitamin D on the activity of $\beta$-catenin in the endometrium of women with endometriosis: a randomized exploratory trial. Gynecol Endocrinol. 2021. 37(3): 278-282.

[17] Karamian A, Paktinat S, Esfandyari S, et al. Pyrvinium pamoate induces in-vitro suppression of IL-6 and IL-8 produced by human endometriotic stromal cells. Hum Exp Toxicol. 2021. 40(4): 649-660.

[18] Zhu X, Li Y, Zhou R, Wang N, Kang S. Knockdown of E-cadherin expression of endometrial epithelial cells may activate Wnt/ $\beta$-catenin pathway in vitro. Arch Gynecol Obstet. 2018. 297(1): 117-123.

[19] Li J, Dai Y, Zhu H, Jiang Y, Zhang S. Endometriotic mesenchymal stem cells significantly promote fibrogenesis in ovarian endometrioma through the Wnt/ $\beta$-catenin pathway by paracrine production of TGF- $\beta 1$ and Wnt1. Hum Reprod. 2016. 31(6): 1224-35.

[20] Zhang H, Li G, Sheng X, Zhang S. Upregulation of miR-33b promotes endometriosis via inhibition of Wnt/ $\beta$-catenin signaling and ZEB1 expression. Mol Med Rep. 2019. 19(3): 2144-2152.

[21] Zhang L, Xiong W, Xiong Y, et al. Intracellular Wnt/Beta-Catenin Signaling Underlying 17betaEstradiol-Induced Matrix Metalloproteinase 9 Expression in Human Endometriosis. Biol Reprod. 2016. 94(3): 70.

[22] Miyazaki K, Dyson MT, Coon V JS, et al. Generation of Progesterone-Responsive Endometrial Stromal Fibroblasts from Human Induced Pluripotent Stem Cells: Role of the WNT/CTNNB1 Pathway. Stem Cell Reports. 2018. 11(5): 1136-1155.

[23] Fontenot E, Rossi E, Mumper R, et al. A novel monoclonal antibody to secreted frizzled-related protein 2 inhibits tumor growth. Mol Cancer Ther. 2013. 12(5): 685-95.

[24] Heinosalo T, Gabriel M, Kallio L, et al. Secreted frizzled-related protein 2 (SFRP2) expression promotes lesion proliferation via canonical WNT signaling and indicates lesion borders in extraovarian endometriosis. Hum Reprod. 2018. 33(5): 817-831. 
[25] Gabriel M, Fey V, Heinosalo T, et al. A relational database to identify differentially expressed genes in the endometrium and endometriosis lesions. Sci Data. 2020. 7(1): 284.

[26] Cai C, Wang W, Tu Z. Aberrantly DNA Methylated-Differentially Expressed Genes and Pathways in Hepatocellular Carcinoma. J Cancer. 2019. 10(2): 355-366.

[27] Rahmioglu N, Drong AW, Lockstone $\mathrm{H}$, et al. Variability of genome-wide DNA methylation and mRNA expression profiles in reproductive and endocrine disease related tissues. Epigenetics. 2017. 12(10): 897908.

[28] Yotova I, Hsu E, Do C, et al. Epigenetic Alterations Affecting Transcription Factors and Signaling Pathways in Stromal Cells of Endometriosis. PLoS One. 2017. 12(1): e0170859.

[29] Chen H, Malentacchi F, Fambrini M, Harrath AH, Huang H, Petraglia F. Epigenetics of Estrogen and Progesterone Receptors in Endometriosis. Reprod Sci. 2020. 27(11): 1967-1974.

[30] Koninckx PR, Ussia A, Adamyan L, Wattiez A, Gomel V, Martin DC. Pathogenesis of endometriosis: the genetic/epigenetic theory. Fertil Steril. 2019. 111(2): 327-340.

[31] Borghese B, Zondervan KT, Abrao MS, Chapron C, Vaiman D. Recent insights on the genetics and epigenetics of endometriosis. Clin Genet. 2017. 91(2): 254-264.

[32] Esfandiari F, Favaedi R, Heidari-Khoei $\mathrm{H}$, et al. Insight into epigenetics of human endometriosis organoids: DNA methylation analysis of HOX genes and their cofactors. Fertil Steril. 2021. 115(1): 125137.

[33] Maniglio P, Ricciardi E, Laganà AS, Triolo O, Caserta D. Epigenetic modifications of primordial reproductive tract: A common etiologic pathway for Mayer-Rokitansky-Kuster-Hauser Syndrome and endometriosis. Med Hypotheses. 2016. 90: 4-5.

[34] Pazhohan A, Amidi F, Akbari-Asbagh F, et al. The Wnt/ $\beta$-catenin signaling in endometriosis, the expression of total and active forms of $\beta$-catenin, total and inactive forms of glycogen synthase kinase3 $\beta$, WNT7a and DICKKOPF-1. Eur J Obstet Gynecol Reprod Biol. 2018. 220: 1-5.

[35] Fan J, Zhang Y, Mu J, et al. TET1 exerts its anti-tumor functions via demethylating DACT2 and SFRP2 to antagonize $\mathrm{Wnt} / \beta$-catenin signaling pathway in nasopharyngeal carcinoma cells. Clin Epigenetics. 2018. 10(1): 103.

[36] Yang M, Jiang C, Chen H, Nian Y, Bai Z, Ha C. The involvement of osteopontin and matrix metalloproteinase- 9 in the migration of endometrial epithelial cells in patients with endometriosis. Reprod Biol Endocrinol. 2015. 13: 95.

[37] Klemmt P, Starzinski-Powitz A. Molecular and Cellular Pathogenesis of Endometriosis. Curr Womens Health Rev. 2018. 14(2): 106-116. 
[38] Houshdaran S, Oke AB, Fung JC, Vo KC, Nezhat C, Giudice LC. Steroid hormones regulate genomewide epigenetic programming and gene transcription in human endometrial cells with marked aberrancies in endometriosis. PLoS Genet. 2020. 16(6): e1008601.

[39] Dyson MT, Roqueiro D, Monsivais D, et al. Genome-wide DNA methylation analysis predicts an epigenetic switch for GATA factor expression in endometriosis. PLoS Genet. 2014. 10(3): e1004158.

[40] Ji F, Yang X, He Y, Wang H, Aili A, Ding Y. Aberrant endometrial DNA methylome of homeobox A10 and catechol-O-methyltransferase in endometriosis. J Assist Reprod Genet. 2017. 34(3): 409-415.

[41] Barjaste N, Shahhoseini M, Afsharian P, Sharifi-Zarchi A, Masoudi-Nejad A. Genome-wide DNA methylation profiling in ectopic and eutopic of endometrial tissues. J Assist Reprod Genet. 2019. 36(8): 1743-1752.

[42] Anastas JN, Moon RT. WNT signalling pathways as therapeutic targets in cancer. Nat Rev Cancer. 2013. 13(1): 11-26.

\section{Tables}

Tab. $1 \mathrm{GO}$ analysis of the low methylation expression gene from the GSE47359 data

\begin{tabular}{lllll} 
Category & Term & Count & PValue \\
\hline GOTERM_CC_DIRECT & GO:0000790 nuclear chromatin & 4 & 0.005055271 \\
\hline GOTERM_BP_DIRECT & GO:0042493 response to drug & 3 & 0.011057299 \\
\hline GOTERM_BP_DIRECT & $\begin{array}{l}\text { GO:0031668 cellular response to extracellular } \\
\text { stimulus }\end{array}$ & 2 & 0.029690877 \\
\hline GOTERM_MF_DIRECT & GO:0003713 transcription coactivator activity & 3 & 0.030570734 \\
\hline GOTERM_BP_DIRECT & $\begin{array}{l}\text { GO:0048646 anatomical structure formation } \\
\text { involved in morphogenesis }\end{array}$ & 2 & 0.031939077 \\
\hline GOTERM_BP_DIRECT & $\begin{array}{l}\text { GO:0045944 positive regulation of transcription } \\
\text { from RNA polymerase II promoter }\end{array}$ & 5 & 0.043516788 \\
\hline GOTERM_CC_DIRECT & GO:0005667 transcription factor complex & 3 & 0.053312753 \\
\hline GOTERM_CC_DIRECT & $\begin{array}{l}\text { GO:0090575 RNA polymerase II transcription } \\
\text { factor complex }\end{array}$ & 2 & 0.058856003 \\
\hline GOTERM_BP_DIRECT & $\begin{array}{l}\text { GO:0050680 negative regulation of epithelial cell } \\
\text { proliferation }\end{array}$ & 2 & 0.099183706 \\
\hline GOTERM_BP_DIRECT & GO:0035914 skeletal muscle cell differentiation & 2 & 0.099183706
\end{tabular}


After the $\mathrm{GO}$ analysis of the low methylation expression , the functions of these hypomethylated genes were explored in several important cell processes, including repressor, secreted, and signaling (Tab. 1).

Tab 2 GO analysis of the upregulation genes from the GSE135485 data 


\begin{tabular}{|c|c|c|c|}
\hline Category & Term & Count & PValue \\
\hline GOTERM_BP_DIRECT & GO:0008584 male gonad development & 7 & 1.35E-07 \\
\hline GOTERM_MF_DIRECT & $\begin{array}{l}\text { GO:0000980 RNA polymerase II distal enhancer } \\
\text { sequence-specific DNA binding }\end{array}$ & 4 & $5.23 E-04$ \\
\hline GOTERM_BP_DIRECT & GO:0008585 female gonad development & 3 & $6.58 \mathrm{E}-04$ \\
\hline GOTERM_MF_DIRECT & GO:0043565 sequence-specific DNA binding & 7 & 0.001463497 \\
\hline GOTERM_MF_DIRECT & $\begin{array}{l}\text { GO:0001077 transcriptional activator activity, } \\
\text { RNA polymerase II core promoter proximal region } \\
\text { sequence-specific binding }\end{array}$ & 5 & 0.002513506 \\
\hline GOTERM_BP_DIRECT & $\begin{array}{l}\text { GO:0006366 transcription from RNA polymerase } \\
\text { Il promoter }\end{array}$ & 6 & 0.009651672 \\
\hline GOTERM_BP_DIRECT & $\begin{array}{l}\text { GO:0045944 positive regulation of transcription } \\
\text { from RNA polymerase II promoter }\end{array}$ & 8 & 0.01158106 \\
\hline GOTERM_MF_DIRECT & G0:0016491 oxidoreductase activity & 4 & 0.012548298 \\
\hline GOTERM_BP_DIRECT & GO:0007584 response to nutrient & 3 & 0.015396063 \\
\hline GOTERM_BP_DIRECT & $\begin{array}{l}\text { Go:0050810 regulation of steroid biosynthetic } \\
\text { process }\end{array}$ & 2 & 0.020307471 \\
\hline GOTERM_MF_DIRECT & $\begin{array}{l}\text { G0:0047498 calcium-dependent phospholipase } \\
\text { A2 activity }\end{array}$ & 2 & 0.021652815 \\
\hline GOTERM_BP_DIRECT & $\begin{array}{l}\text { GO:0036149 phosphatidylinositol acyl-chain } \\
\text { remodeling }\end{array}$ & 2 & 0.040211948 \\
\hline GOTERM_BP_DIRECT & $\begin{array}{l}\text { GO:0036148 phosphatidylglycerol acyl-chain } \\
\text { remodeling }\end{array}$ & 2 & 0.045126018 \\
\hline GOTERM_BP_DIRECT & $\begin{array}{l}\text { G0:0036150 phosphatidylserine acyl-chain } \\
\text { remodeling }\end{array}$ & 2 & 0.045126018 \\
\hline GOTERM_BP_DIRECT & $\begin{array}{l}\text { GO:0036152 phosphatidylethanolamine acyl- } \\
\text { chain remodeling }\end{array}$ & 2 & 0.059721243 \\
\hline GOTERM_BP_DIRECT & GO:0050482 arachidonic acid secretion & 2 & 0.059721243 \\
\hline GOTERM_MF_DIRECT & GO:0008270 zinc ion binding & 7 & 0.0613717 \\
\hline GOTERM_BP_DIRECT & $\begin{array}{l}\text { GO:0036151 phosphatidylcholine acyl-chain } \\
\text { remodeling }\end{array}$ & 2 & 0.066936914 \\
\hline GOTERM_MF_DIRECT & G0:0003682 chromatin binding & 4 & 0.068885928 \\
\hline GOTERM_BP_DIRECT & $\begin{array}{l}\text { GO:0009755 hormone-mediated signaling } \\
\text { pathway }\end{array}$ & 2 & 0.069330097 \\
\hline GOTERM_MF_DIRECT & GO:0004623 phospholipase A2 activity & 2 & 0.072674345 \\
\hline GOTERM_MF_DIRECT & GO:0017147 Wnt-protein binding & 2 & 0.072674345 \\
\hline
\end{tabular}




\begin{tabular}{llll} 
GOTERM_BP_DIRECT & $\begin{array}{l}\text { GO:0070374 positive regulation of ERK1 and } \\
\text { ERK2 cascade }\end{array}$ & 3 & 0.073862462 \\
\hline GOTERM_CC_DIRECT & $\begin{array}{l}\text { G0:0090575 RNA polymerase II transcription } \\
\text { factor complex }\end{array}$ & 2 & 0.07673751 \\
\hline GOTERM_BP_DIRECT & G0:0050873 brown fat cell differentiation & 2 & 0.078843017 \\
\hline GOTERM_MF_DIRECT & $\begin{array}{l}\text { G0:0003700 transcription factor activity, } \\
\text { sequence-specific DNA binding }\end{array}$ & 6 & 0.081625912 \\
\hline GOTERM_MF_DIRECT & $\begin{array}{l}\text { G0:0004879 RNA polymerase II transcription } \\
\text { factor activity, ligand-activated sequence-specific } \\
\text { DNA binding }\end{array}$ & 2 & 0.083902905 \\
\hline GOTERM_BP_DIRECT & $\begin{array}{l}\text { GO:0006654 phosphatidic acid biosynthetic } \\
\text { process }\end{array}$ & 2 & 0.085915314 \\
\hline GOTERM_CC_DIRECT & G0:0005576 extracellular region & 8 & 0.08929768 \\
\hline GOTERM_BP_DIRECT & G0:0035094 response to nicotine & 2 & 0.090600685 \\
\hline GOTERM_BP_DIRECT & $\begin{array}{l}\text { G0:0030522 intracellular receptor signaling } \\
\text { pathway }\end{array}$ & 2 & 0.092934565 \\
\hline GOTERM_BP_DIRECT & $\begin{array}{l}\text { G0:0010811 positive regulation of cell-substrate } \\
\text { adhesion }\end{array}$ & 2 & 0.092934565 \\
\hline GOTERM_BP_DIRECT & G0:0048468 cell development & 2 & 0.097584789
\end{tabular}

The significant terms of GO enrichment analysis performed by DAVID from the GSE135485 data.

\section{Figures}




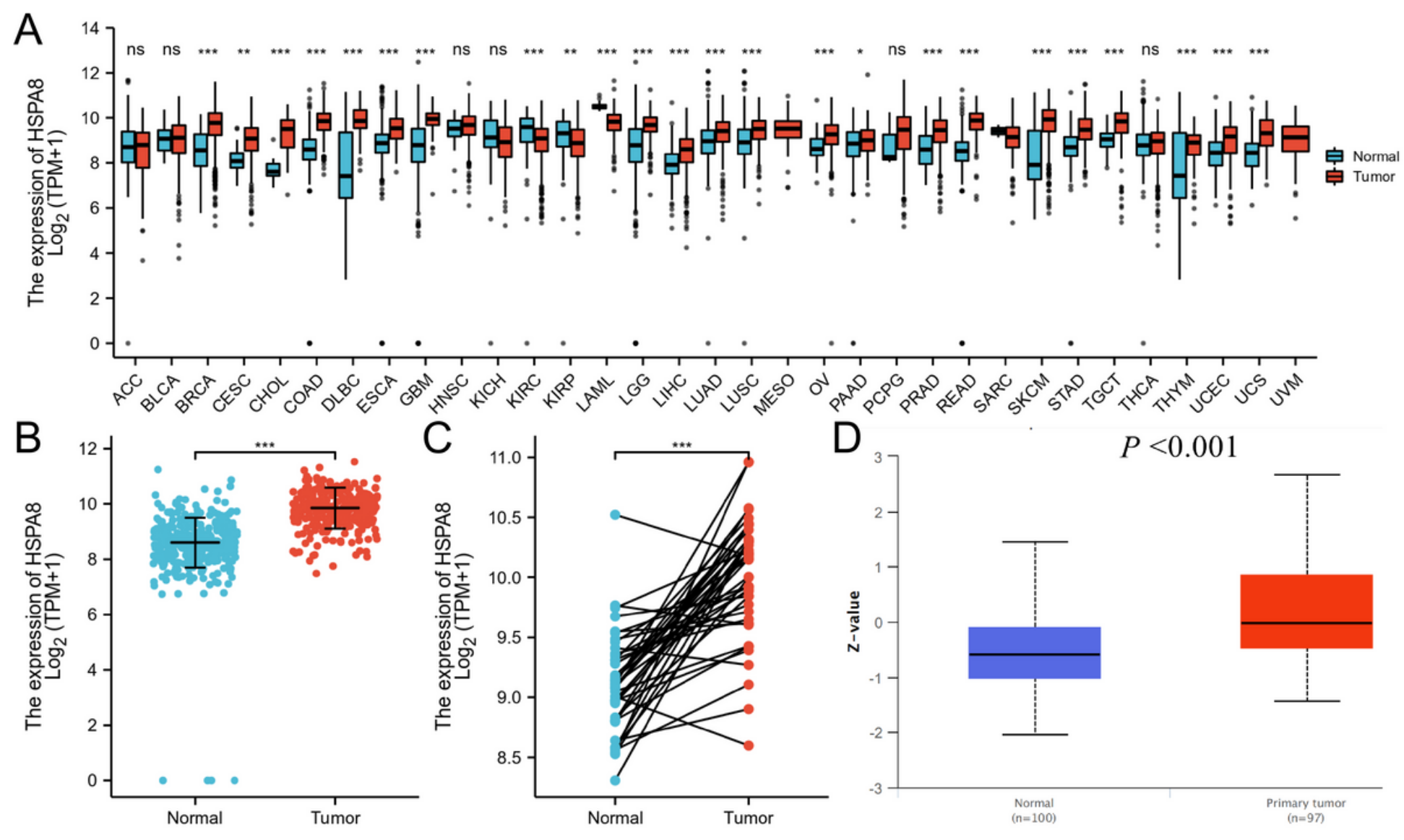

Figure 1

Heatmap clustering of the demethylation of the SFRP2 promoter from GSE47359 in 3 EMS samples vs 6 normal endometrium tissue samples. 

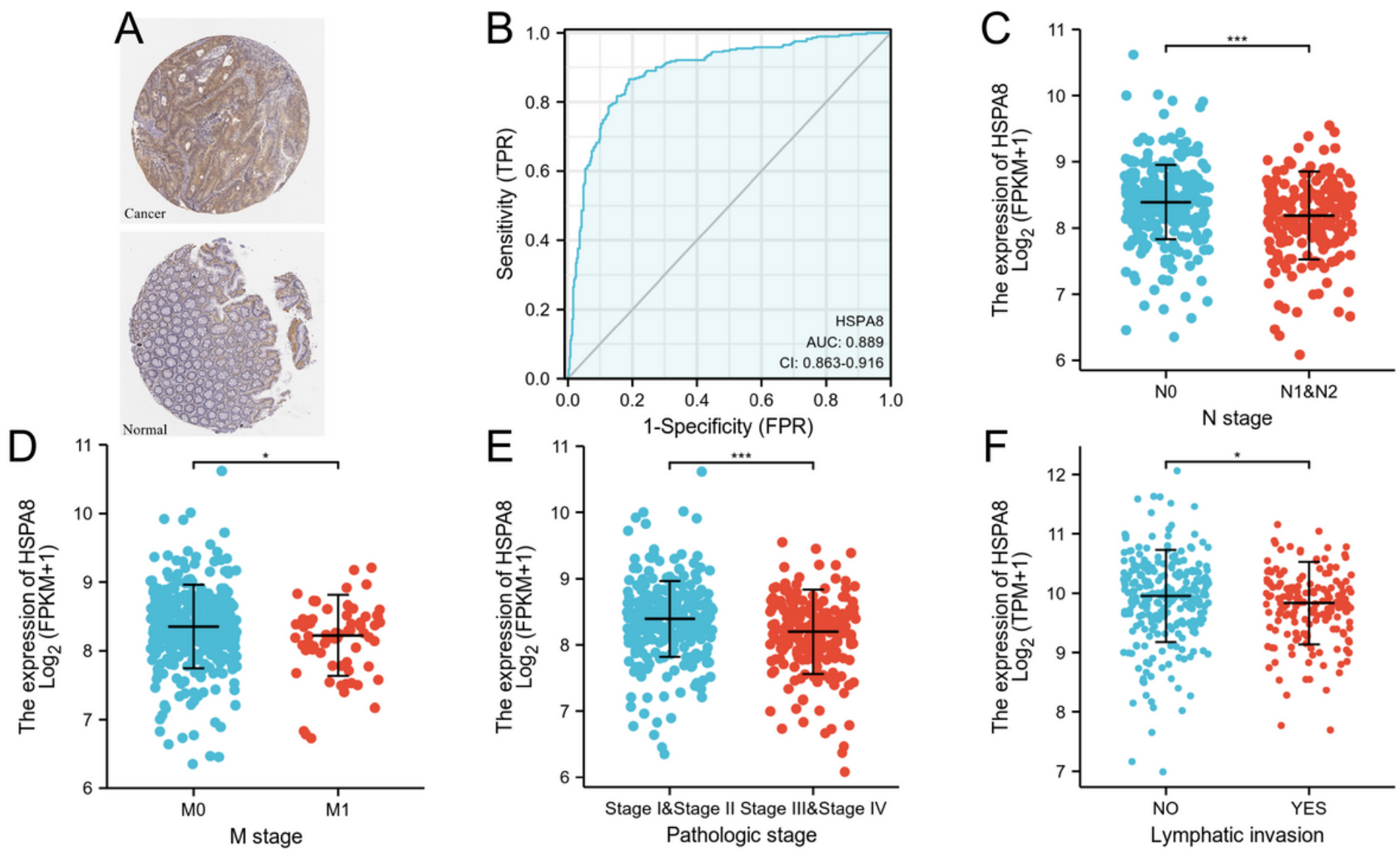

Figure 2

Heatmap clustering of differentially expressed genes in mRNA expression profiling datasets (GSE135485), which includes 54 EMS samples and 4 normal endometrium tissue samples.
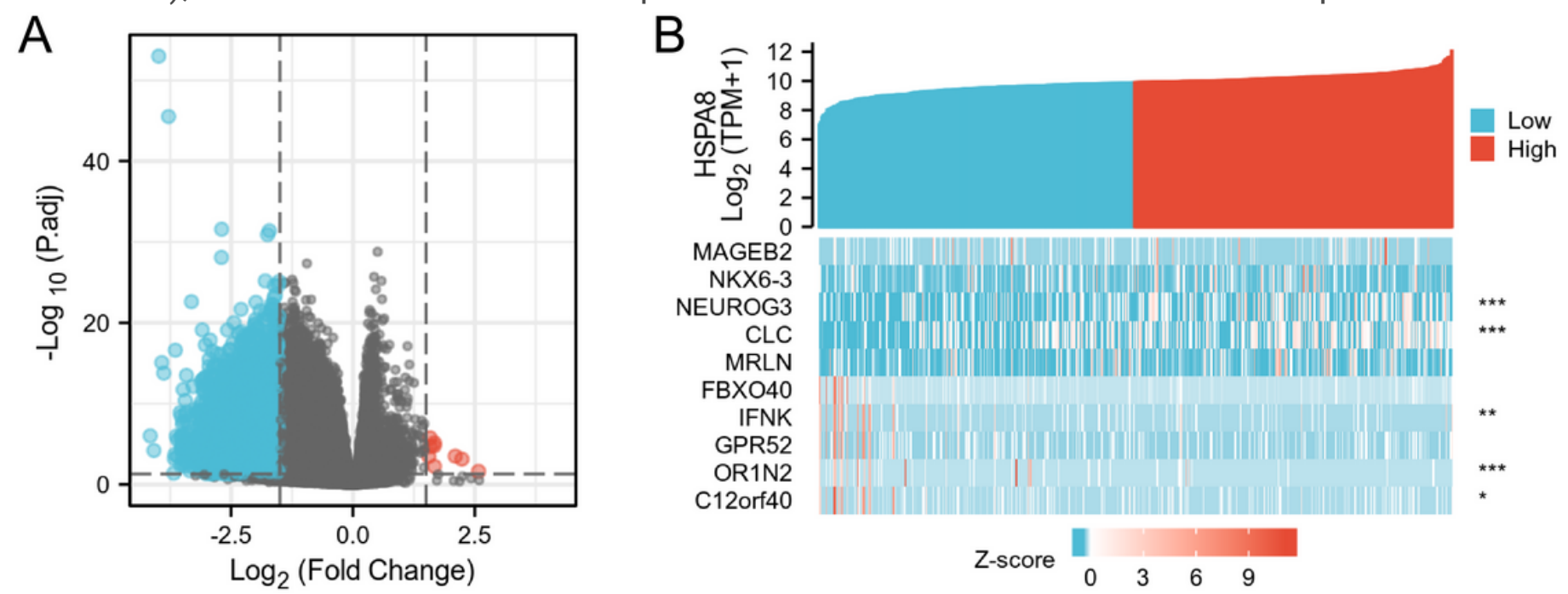

\section{Figure 3}

Identification of aberrantly methylated-differentially expressed genes in mRNA expression profiling datasets (GSE135485) and gene methylation profiling datasets (GSE47359). 

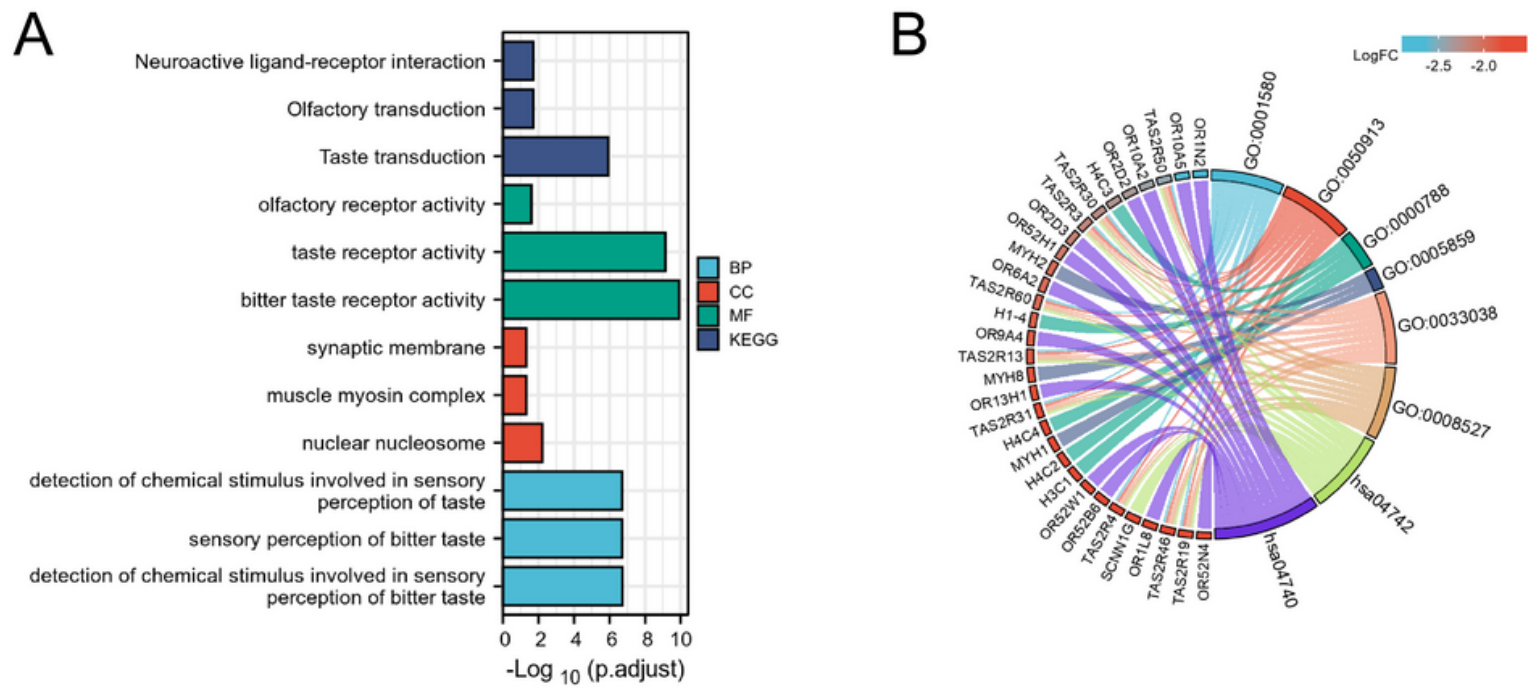

\section{Figure 4}

Immunohistochemical staining of SFRP2 protein in different endometrium tissues. A Normal endometrium B Eutopic endometrium (endometrium in the uterine cavity of the EMS patients) C ectopic endometrium D Through the immunohistochemistry staining, it was found that SFRP2 was clearly located in the cytoplasm and was yellowy-brown. 
A

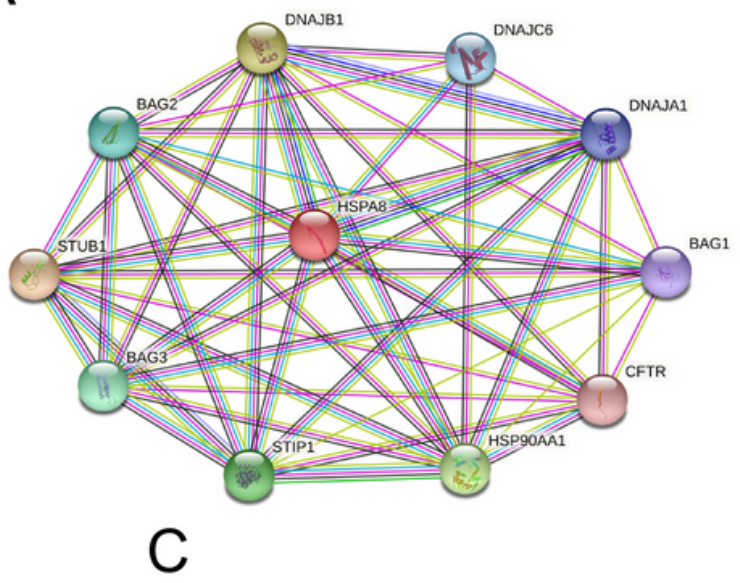

B

\begin{tabular}{ccc} 
Gene symbol & Annotation & Score \\
\hline STUB1 & E3 ubiquitin-protein ligase CHIP & 0.999 \\
DNAJB1 & DnaJ homolog subfamily B member 1 & 0.999 \\
HSP90AA1 & Heat shock protein HSP 90-alpha & 0.999 \\
STIP1 & Stress-induced-phosphoprotein 1 & 0.999 \\
BAG3 & BAG family molecular chaperone regulator 3 & 0.999 \\
BAG2 & BAG family molecular chaperone regulator 2 & 0.999 \\
DNAJC6 & Putative tyrosine-protein phosphatase auxilin & 0.999 \\
DNAJA1 & DnaJ homolog subfamily A member 1 & 0.999 \\
BAG1 & BAG family molecular chaperone regulator 1 & 0.999 \\
CFTR & Cystic fibrosis transmembrane conductance regulator & 0.998 \\
\hline
\end{tabular}

PSG4 PIWIL3 GALR1 TMEM212

PSG1 RNF17 SALL3 3 PATE1

PAPOLB

PRB4 BOLL

$\mathrm{PRH} 2$

RAB40AL
TMPRSS12 OR52B6

TRPC5
C12Orf40 MROH9 HIST1H1E

$\begin{array}{lllll}\text { C8A } & \text { C9Orf } 31 & \text { SCGB1A1 } & \text { KRT14 } & \text { HIST1H4F } \\ \text { LHFPL4 } & \text { SFTPC } & \text { KRT31 }\end{array}$

FLG2 CHST9 TMPRSS11A EPGN

PLIN4 ACADL

TRHR

GNRHR LHCGR ERICH3 INHA

LRRTM

ZIC4 GABRG3 NPAP1

XAGE2B LHX8

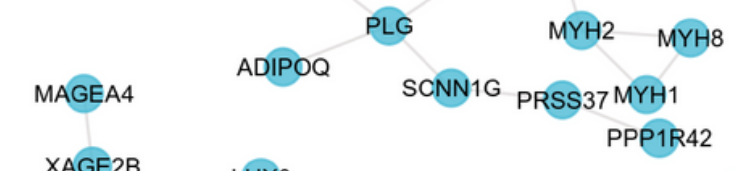

PPP1R42

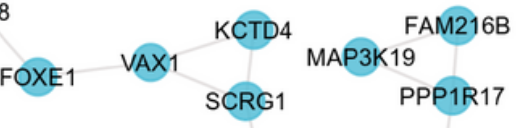

CYP2A6

CYP4A22 CRCT1 PNLIPR S 3 3 RN1

ERBB4 4 PAGE4 REC114 CODC83 IFNK

SLC6A2 CAV3 ASB15 ANKRD30BL

HR2C TRDN CSRP3

GPR112 ${ }^{\text {TENM1 VWC2L }}$

GPR22 ${ }_{\text {GPR12 }}^{\mathrm{CDH} 9}$ FBX040

GPR52

\section{Figure 5}

Increased SFRP2 expression in EEECs and endometriosis tissues. A Western blot analysis of SFRP2 protein level in EMS vs. NE(Normal endometrium) B Western blot analysis on SFRP2 protein level in EEECs vs. NEECs C Western blot data of SFRP2 protein level in EMS vs. NE D Western blot data on SFRP2 protein level. in EEECs vs. NEECs E Real-time RT PCR. The mRNA expression levels of SFRP2 in EEECs vs NEECs. F Real-time RT PCR. Compared with the normal endometrium, The mRNA expression levels of SFRP2 in EMS vs Normal endometrium. 


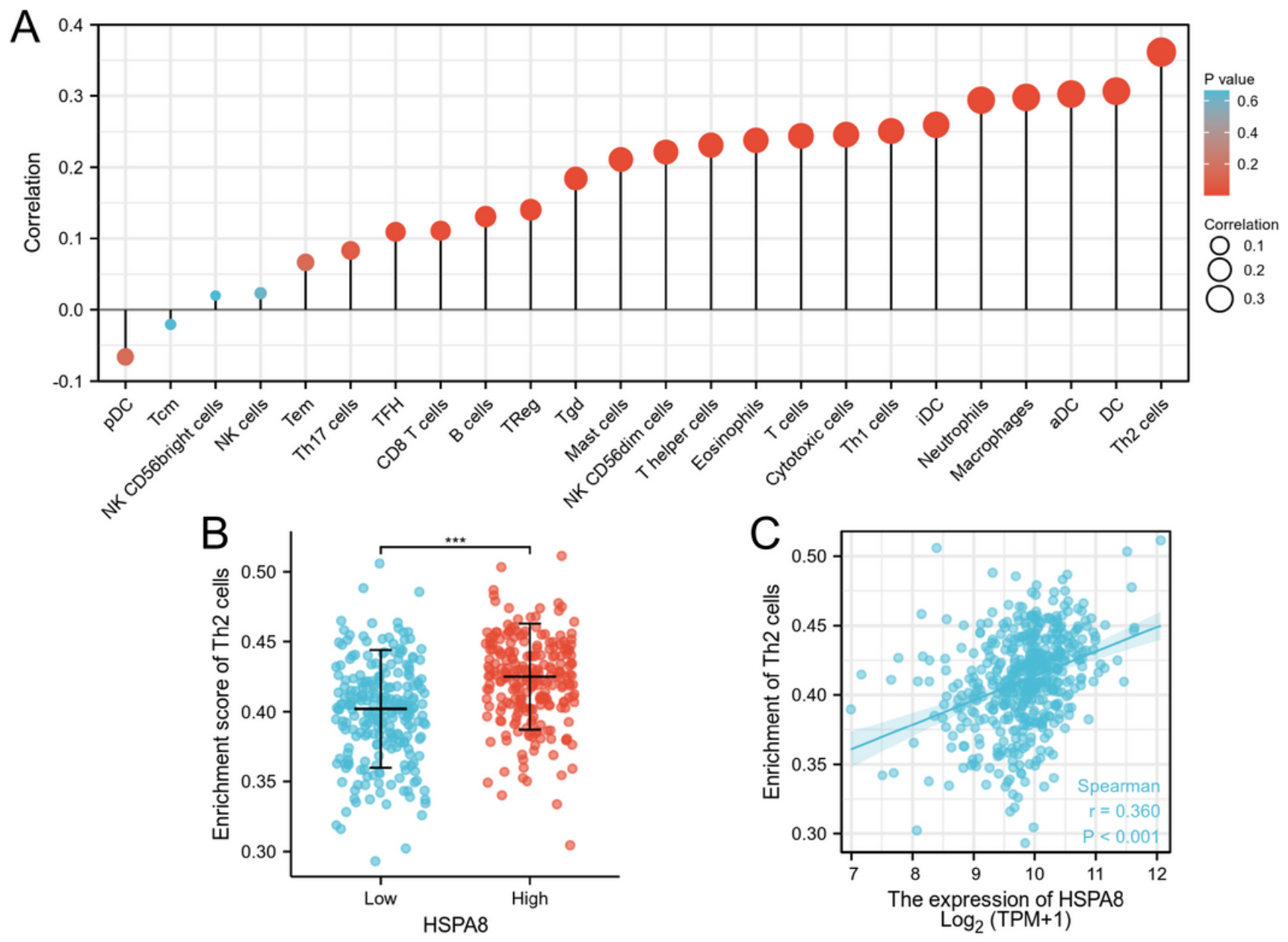

Figure 6

Demethylation of the SFRP2 promoter in endometriosis A MSP. The methylation rates of normal endometrium and EMS endmetrium were detected by MSP respectively. B Bisulfite sequencing PCR on SFRP2 promoter in EEECs and NEECs. C MSP. EEECs were grown and treated with 5-Aza and subjected to MSP. D Real-time RT PCR. EEECs were grown and treated with 5-Aza and subjected to Real-time RT PCR E Real-time RT PCR. EEECs were grown and transfected with DNMT1 shRNAs and subjected to Real-time RT PCR. F Western blot analysis. EEECs were grown and treated with 5-Aza and subjected to Western blot analysis. G Western blot analysis. EEECs were grown and transfected with DNMT1 shRNAs and subjected to Western blot. 


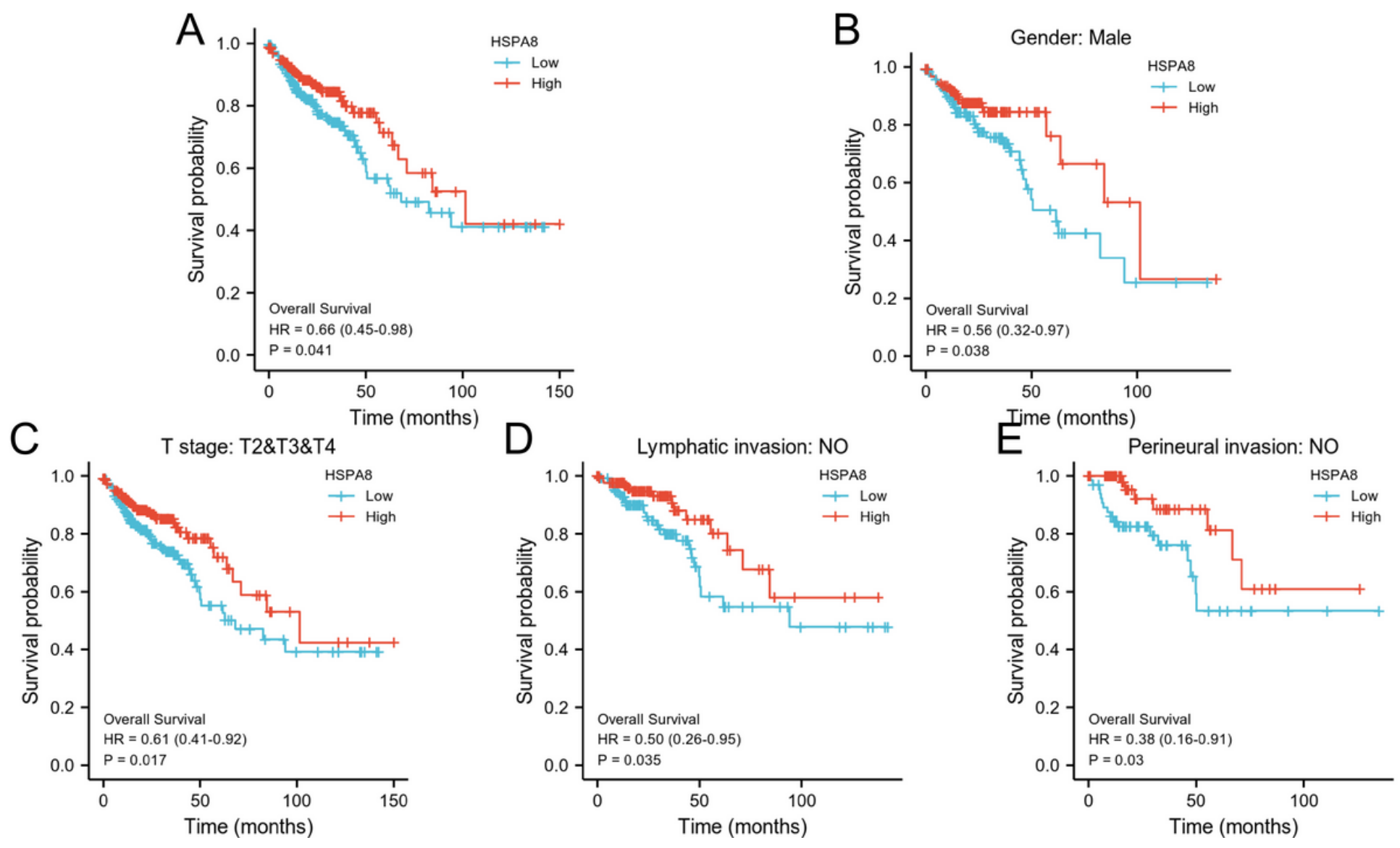

Figure 7

Increase of Wnt signaling gene expression and activity after ectopic SFRP2 expression in EEECs. A Realtime RT PCR. EEECs were grown and transfected with SFRP2 CDNA and subjected to Real-time RT PCR. B Luciferase reporter assay. EEECs were grown and transfected with SFRP2 cDNA and subjected to the Luciferase reporter assay. C Real-time RT PCR. EEECs were grown and transfected with SFRP2 CDNA and subjected to Real-time RT PCR. D Western blot analysis. EEECs were grown and transfected with SFRP2 cDNA and subjected to Western blot analysis 